\title{
Smoking behavior and smoking index as prognostic indicators for patients with esophageal squamous cell carcinoma who underwent surgery: A large cohort study in Guangzhou, China
}

\author{
Lili Liu ${ }^{1,2,3 *}$, Chaoyun Huang ${ }^{1,2,3 *}$, Wei Liao ${ }^{1,2,4 *}$, Shuwei Chen ${ }^{1,2,5}$, Shaohang Cai ${ }^{1,2,4,6}$
}

\begin{abstract}
INTRODUCTION This study aimed to evaluate the association between smoking and smoking index with clinical outcomes of esophageal squamous cell carcinoma patients.

METHODS This is a retrospective analysis conducted on consecutive patients with esophageal carcinoma who underwent esophagectomy from January 2005 to December 2010. All patients had pathologically confirmed esophageal squamous cell carcinoma. The association between smoking and sociodemographic characteristics with overall survival and disease-free survival was analyzed. Serum carcinoembryonic antigen was measured using an electrochemiluminescence immunoassay.

RESULTS A total of 944 patients were enrolled. Kaplan-Meier analysis indicated that esophageal squamous cell carcinoma patients who smoked had a significantly worse prognosis in terms of both overall survival $(p=0.007)$ and disease-free survival $(p=0.010)$. Multivariate analysis demonstrated that age $(\mathrm{p}=0.001)$, carcinoembryonic antigen $(\mathrm{p}=0.012)$, tumor-node-metastasis (TNM) staging $(\mathrm{p}<0.001)$ and smoking $(\mathrm{p}=0.048)$ were independently correlated with overall survival, while only TNM stage $(p<0.001)$ and smoking $(\mathrm{p}=0.041)$ were identified as independent factors of disease-free survival. We divided the smoking population into two groups (smoking index $<400$ and $\geq 400$ ). Kaplan-Meier survival analysis indicated that a smoking index $<400$ was associated with a significantly better prognosis in terms of both overall survival $(p=0.003)$ and favorable disease-free survival $(p=0.032)$. Multivariate analysis showed that age $(\mathrm{p}<0.001)$, TNM staging $(\mathrm{p}<0.001)$, and smoking index $(\mathrm{p}=0.025)$ were independent factors of overall survival, whereas for disease-free survival, only TNM stage $(\mathrm{p}=0.001)$ and smoking index $(\mathrm{p}=0.025)$ were identified.

CONCLUSIONS Overall survival was significantly associated with smoking in esophageal squamous cell carcinoma patients. For esophageal squamous cell carcinoma patients who smoke, a higher smoking index is associated with worse clinical outcomes. Therefore, smoking may be used as a predictive indicator for pretreatment evaluation and adjustment of treatment regimen.
\end{abstract}

\section{AFFILIATION \\ 1 State Key Laboratory of Oncology in South China, Guangzhou, China 2 Collaborative Innovation Center for Cancer Medicine, Guangzhou, China \\ 3 Department of Pathology, Sun Yat-sen University Cancer Center, Guangzhou, China 4 Intensive Care Unit, Sun Yat- sen University Cancer Center, Guangzhou, China \\ 5 Department of Head and Neck Surgery, Sun Yat-sen University Cancer Center, Guangzhou, China 6 Department of Infectious Diseases and Hepatology Unit, Nanfang Hospital, Southern Medical University, Guangzhou, China \\ ${ }^{*}$ Contributed equally}

\section{CORRESPONDENCE TO}

Shaohang Cai. Department of Infectious Diseases and Hepatology Unit, Nanfang Hospital, Southern Medical University, No. 1838, Guangzhou North Avenue, Guangzhou 510515, China. E-mail: shaohangcai@foxmail.com

Shuwei Chen. Department of Head and Neck Surgery, Sun Yat-sen University Cancer Center, No. 651 Dongfeng East Road, Guangzhou 510060, China. E-mail: chenshuw@sysucc.org.cn

\section{KEYWORDS}

smoking, overall survival, esophageal squamous cell carcinoma, prognostic marker

Received: 21 October 2019

Revised: 24 January 2020

Accepted: 25 January 2020 


\section{INTRODUCTION}

Esophageal cancer (EC) is a common malignancy worldwide $^{1}$. According to the International Agency for Research on Cancer, EC is one of the eight most common malignancies ${ }^{2,3}$. EC is also one of the six most deadliest tumors in the world ${ }^{2,3}$. China has a high risk of EC, and the mortality rate of EC ranks fourth in all malignant tumors, followed by lung cancer, stomach cancer, and liver cancer ${ }^{4,5}$. EC consists of two primary pathological types: squamous cell carcinoma and adenocarcinoma. Worldwide, the incidence of esophageal squamous cell carcinoma (ESCG) is slightly higher than that of adenocarcinoma. Between these two types of cancer, adenocarcinoma mainly occurs in Western countries, while in East Asian countries and China, EC is primarily squamous cell carcinoma ${ }^{5}$.

The pathogenesis of EC is complex and not fully understood. Epidemiological studies have confirmed that smoking is an important risk factor of $\mathrm{ESCC}^{6,7}$. The Center for International Cancer Research categorizes tobacco and alcohol as Class I carcinogens with sufficient evidence to prove their carcinogenicity. Several studies have shown that smoking is an important risk factor for the development of EC, especially ESCC $^{8-10}$. Zambon et al. ${ }^{11}$ conducted a case-control study on the relationship between EC and smoking in three regions of northern Italy, involving 275 patients with ESCC and 593 control. They found that smoking was a strong risk factor for the development of EC. The incidence of ESCG was 7 times higher than that of non-smokers in the study population ${ }^{11}$.

One of the most common ways to model smoking is by dividing subjects into never and current smoker categories ${ }^{12}$. Compared to never smoking, current smoking was associated with increased cardiovascular disease risk $^{13}$. However, one way that heterogeneity can enter into smoking status categories is via smoking amount, specifically cigarettes per day. Previous studies suggest that there is an increased risk of death due to coronary heart disease with increased amount ${ }^{14}$. This is a common way of adjusting for smoking ${ }^{12}$. Pack-years is a cumulative measure of smoking and is generally calculated by multiplying average packs smoked per day by the duration of smoking in years ${ }^{12}$. Similar to pack-years, we used the smoking index, which integrates the duration of smoking and the number of cigarettes smoked per day. Thus, the smoking index is equal to the daily tobacco intake multiplied by the duration of smoking in years.

Tobacco has been confirmed to be associated with the occurrence of many tumors ${ }^{15,16}$. Tobacco smoking is well known to promote the development of EC, irrespective of the pathological type ${ }^{17}$. A previous review noted that tobacco smoking induces a more malignant tumor phenotype by increasing the cell proliferation, cell mobility, as well as angiogenesis, and by activating cellular pro-survival pathways ${ }^{18}$. However, the role of smoking in the prognosis of ESCC still lacks evidence. Studies have also found that tobacco induces increased serum carcinoembryonic antigen (CEA). CEA is a promising tumor biomarker in patients with ESCC. Studies have indicated that CEA is a predictor of OS for prognosis in ESCC $^{19,20}$.

Patients with esophageal squamous cell cancer usually have a poor prognosis. Despite the various advances in multimodal treatment strategies, the 5 -year overall survival (OS) rate is still poor ${ }^{1}$. In addition, because of differences among patients of different ethnic and geographical origins, the association and predictive value of smoking need to be tested in different populations. Hence, the current study aims to determine the association of smoking with OS in ESCC patients.

\section{METHODS}

\section{Patient selection and data collection}

This is a retrospective analysis conducted on consecutive patients with EC who underwent esophagectomy at Sun Yat-sen University Cancer Center, Guangzhou, China, between January 2005 and December 2010. The study was approved by the Medical Ethics Committee and Clinical Trial Review Committee of the Cancer Center. All procedures followed were in accordance with the ethical standards of the responsible committee on human experimentation and with the Helsinki Declaration of 1975 , as revised in 2008. Informed consent was obtained from all patients for inclusion in the study.

A total of 944 patients were enrolled. All patients had pathologically confirmed ESCC. Patients were excluded if: 1) they had an additional carcinoma, 2) they underwent palliative esophagectomy, or 3) their clinical data were not complete. All data were collected 
from medical records, and survival data were obtained from the Cancer Center's follow-up registry. The pathologic staging of tumors for patients was based on the 8th edition of the American Joint Committee on Cancer (AJCC) tumor-node-metastasis classification ${ }^{6}$. In our study, all patient information was extracted from medical records. To control for bias, patients were included in the study consecutively. We included all patients with complete data in the analysis. The flow chart is shown in Supplementary file Figure 1.

\section{Study endpoints}

In this study, the primary endpoint was disease-free survival (DFS), and the secondary endpoint was OS. DFS was defined from the date of surgery to the date of disease locoregional relapse or distant metastasis or death from any other cause. OS was defined as the interval from the date of surgery to the date of death from any cause.

\section{Laboratory tests}

Serum carcinoembryonic antigen (CEA) was measured using a commercially available electrochemiluminescence immunoassay (Cobas E602-2, Hoffmann-La Roche Ltd, Pleasanton, CA, USA). The normal values of CEA are $<5 \mathrm{ng} / \mathrm{mL}$. Serum albumin (ALB) and globulin (GLB) levels were determined using automated techniques (LABOSPECT 008, Hitachi-hitec Globe Ltd, Tokyo, Japan). The normal ALB and GLB levels are 40-55 $\mathrm{g} / \mathrm{L}$ and $20-30 \mathrm{~g} / \mathrm{L}$, respectively.

The tumor size and differentiation were reported by at least three experienced pathologists who were not informed about the patients' preoperative conditions.

\section{Sociodemographic information}

Sociodemographic and smoking characteristics such as gender, age, duration of smoking, daily tobacco intake, and family history, were collected from all participating subjects by a questionnaire. The smoking index integrates the duration of smoking and the number of cigarettes smoked per day, similar to pack-years.

Smoking index $=$ daily tobacco intake $\times$ duration of smoking.

All subjects finished the questionnaire surveys in a quiet room without any interference or disruptions. Professional staff members were available to answer ques-tions if any problems occurred in understanding the survey questions ${ }^{21}$.

To further explore the relationship between different amounts of smoking and clinical features, we divided the smoking population into two groups, those with a smoking index $<400$ and those with $\geq 400$.

\section{Statistical analysis}

Categorical variables were calculated using Fisher's exact test and chi-squared test, while continuous variables were analyzed using Student's t-test. Multivariable logistical regression was performed to assess patient and tumor characteristics. All endpoints were estimated by the Kaplan-Meier method and

Figure 1. Association of smoking index and clinical outcomes in smoking patients: (A) Kaplan-Meier analysis indicated that ESCC patients with smoking were accompanied with significantly worse prognosis in terms of overall survival ( $p=0.007)$; (B) Non-smoking was positively correlated with favorable disease-free survival $(p=0.010)$

A

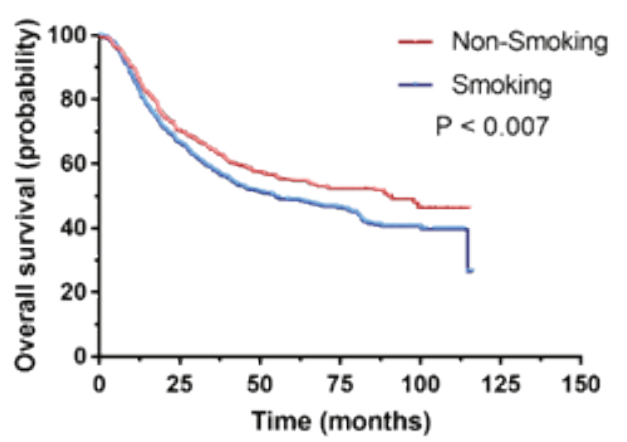

B

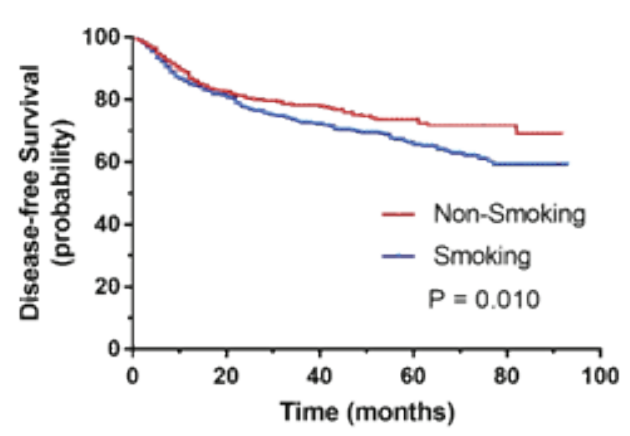


compared using the log-rank test. Multivariable survival analyses were performed using the Cox proportional hazards model to identify important prognostic factors for OS and DFS. Two-sided p-values of $<0.05$ were considered statistically significant. All analyses were performed using SPSS20.0 software (SPSS Inc., Chicago, IL, USA).

\section{RESULTS}

\section{Baseline sociodemographic and clinical characteristics}

Consecutive patients with EG who underwent esophagectomy between January 2005 and December 2010 were enrolled $(n=944)$. Patient outcomes were recorded from January to December 2015. The detailed baseline characteristics according to smoking behavior are listed in Table 1. Serum GLB levels in ESCC patients who smoked $(26.3 \pm 4.9)$ were significantly lower than in ESCC patients who did not smoke $(28.1 \pm 5.6)(\mathrm{p}<0.001)$. The serum level of CEA in the smoking group $(3.5 \pm 2.8 \mathrm{ng} / \mathrm{mL})$ was

Table 1. Baseline demographic and clinical characteristics of ESCC patients, Guangzhou, 2005$2010(\mathrm{~N}=944)$

\begin{tabular}{|c|c|c|c|}
\hline Characteristics & Smokers & Von-smokers & $p$ \\
\hline Sample size & 608 & 336 & \\
\hline Gender, M/F & $599 / 9$ & $127 / 209$ & $<0.001$ \\
\hline Age (years) & $58.1 \pm 8.76$ & $59.0 \pm 9.57$ & 0.135 \\
\hline$A L B, g / L$ & $42.9 \pm 4.2$ & $43.5 \pm 5.1$ & 0.062 \\
\hline GLB, g/L & $26.3 \pm 4.9$ & $28.1 \pm 5.6$ & $<0.001$ \\
\hline CEA, ng/mL & $3.5 \pm 2.8$ & $2.8 \pm 3.8$ & 0.002 \\
\hline Tumor site, $n$ & & & 0.031 \\
\hline Upper & 64 & 25 & \\
\hline Middle & 359 & 227 & \\
\hline Distal & 185 & 84 & \\
\hline Tumor size, cm & $4.6 \pm 1.9$ & $4.3 \pm 1.8$ & 0.003 \\
\hline Differentiation & & & 0.529 \\
\hline $\mathrm{G} 1$ & 128 & 62 & \\
\hline G2 & 291 & 160 & \\
\hline G3 & 189 & 114 & \\
\hline TNM, n & & & 0.008 \\
\hline Stage 1 & 59 & 41 & \\
\hline Stage 2 & 260 & 173 & \\
\hline Stage 3 & 277 & 119 & \\
\hline Stage 4 & 12 & 3 & \\
\hline
\end{tabular}

Numbers with \pm represent mean \pm SD . significantly higher than in the non-smoking group $(2.8 \pm 3.8 \mathrm{ng} / \mathrm{mL})(\mathrm{p}=0.002)$.

The proportion of ESCC patients in the TNM stage was significantly different between the two groups. In the smoking group: 59, 260, 277 and 12 patients were in stages 1, 2, 3 and 4, respectively. Whereas in the non-smoking group: 41, 173, 119 and 3 patients were in stages $1,2,3$ and 4 , respectively $(p=0.008)$. Details are shown in Table 1.

\section{Association of smoking and clinical outcomes}

To determine the prognostic impact of smoking on ESCG patients, Kaplan-Meier survival analysis was performed. Kaplan-Meier analysis indicated that ESCC patients who smoked had a significantly worse prognosis in terms of OS $(\mathrm{p}=0.007)$. The non-smoking group was positively correlated with favorable DFS $(\mathrm{p}=0.010)$ (Figure 1).

In the smoking group, a total of 147 patients (24.2\%) had post-operative complications, compared with 77 in the non-smoking group (22.9\%). Although the rate of post-operative complications was relatively higher in the smoking group, there was no significant difference between the two groups $(p=0.655)$. The most common complications were anastomotic fistula $(\mathrm{n}=55)$, anastomotic stenosis $(\mathrm{n}=40)$, pneumonia $(n=10)$, and other complications. At the end of enrollment of the study, the smoking group had a mortality rate of $54.7 \%(\mathrm{n}=333)$, significantly higher than the non-smoking group with $47.0 \%(n=158)$ $(p=0.022)$.

\section{Analysis of the independent factors for OS and DFS}

Cox regression analysis was used to determine the associations between clinical features with OS. As shown in Tables 2 and 3, multivariate analysis showed that age (hazard ratio, $\mathrm{HR}=1.018 ; 95 \% \mathrm{CI}$ : 1.008-1.028; $\mathrm{p}=0.001)$, CEA $(\mathrm{HR}=1.033 ; 95 \% \mathrm{CI}$ : 1.007-1.060; $\mathrm{p}=0.012)$, TNM staging $(\mathrm{HR}=1.983$; 95\% CI: 1.714-2.293; p<0.001), and smoking $(\mathrm{HR}=1.214 ; 95 \% \mathrm{CI}: 1.002-1.471 ; \mathrm{p}=0.048)$ were independently correlated with OS (Table 2 ). Similarly, multivariate analysis showed that only TNM stage $(\mathrm{HR}=1.453 ; 95 \%$ CI: $1.208-1.747$; $\mathrm{p}<0.001)$ and smoking $(\mathrm{HR}=1.597$; 95\% CI: 1.009 $2.110 ; \mathrm{p}=0.041)$ were independently correlated with DFS (Table 3). 
Table 2. Analysis of the independent factors for overall survival of all ESCC patients, Guangzhou, 2005-2010 ( $\mathrm{N}=944)$

\begin{tabular}{|c|c|c|c|c|c|c|}
\hline \multirow[t]{2}{*}{ Variables } & \multicolumn{3}{|c|}{ Univariate analysis } & \multicolumn{3}{|c|}{ Vultivariate analysis } \\
\hline & IIR & $95 \% \mathrm{CI}$ & p & IIIR & $95 \% \mathrm{CI}$ & p \\
\hline Gender & 1.113 & $0.816-1.520$ & 0.499 & & & \\
\hline Age & 1.016 & $1.006-1.027$ & 0.002 & 1.018 & $1.008-1.028$ & 0.001 \\
\hline ALB & 0.992 & $0.972-1.013$ & 0.471 & & & \\
\hline GLB & 1.013 & $0.995-1.032$ & 0.161 & & & \\
\hline CEA & 1.034 & $1.008-1.061$ & 0.009 & 1.033 & $1.007-1.060$ & 0.012 \\
\hline Tumor site & 0.976 & $0.835-1.142$ & 0.764 & & & \\
\hline Tumor size & 1.027 & $0.977-1.080$ & 0.299 & & & \\
\hline Differentiation & 1.098 & $0.967-1.245$ & 0.150 & & & \\
\hline TNM & 1.933 & $1.663-2.247$ & $<0.001$ & 1.983 & $1.714-2.293$ & $<0.001$ \\
\hline Smoking index & 1.323 & $1.009-1.735$ & 0.043 & 1.214 & $1.002-1.471$ & 0.048 \\
\hline
\end{tabular}

Table 3. Analysis of the independent factors for disease-free survival of all ESCC patients, Guangzhou, 2005$2010(\mathrm{~N}=944)$

\begin{tabular}{|c|c|c|c|c|c|c|}
\hline \multirow[t]{2}{*}{ Variables } & \multicolumn{3}{|c|}{ Univariate analysis } & \multicolumn{3}{|c|}{ Vultivariate analysis } \\
\hline & $I I R$ & $95 \% \mathrm{CI}$ & p & $I I R$ & $95 \% \mathrm{CI}$ & $p$ \\
\hline Gender & 0.755 & $0.476-1.198$ & 0.233 & & & \\
\hline Age & 0.986 & $0.973-1.001$ & 0.058 & & & \\
\hline ALB & 0.990 & $0.960-1.020$ & 0.493 & & & \\
\hline GLB & 1.002 & $0.977-1.027$ & 0.896 & & & \\
\hline CEA & 1.028 & $0.992-1.065$ & 0.125 & & & \\
\hline Tumor site & 0.868 & $0.702-1.074$ & 0.194 & & & \\
\hline Tumor size & 0.969 & $0.903-1.039$ & 0.377 & & & \\
\hline Differentiation & 1.089 & $0.916-1.296$ & 0.333 & & & \\
\hline TNM & 1.438 & $1.188-1.740$ & $<0.001$ & 1.453 & $1.208-1.747$ & $<0.001$ \\
\hline Smoking index & 1.347 & $1.024-1.964$ & 0.002 & 1.597 & $1.209-2.110$ & 0.001 \\
\hline
\end{tabular}

\section{Factors associated with smoking index}

To further explore the relationship between different amounts of smoking and clinical features, we divided the smoking population into those with a smoking index $<400$ and those with $\geq 400$. For ESCC patients who smoked, the results showed that older age was observed among those with a smoking index $\geq 400$ $(p=0.028)$. The proportion of ESCG patients in the TNM stage was significantly different between the two groups. In the group with a smoking index $<400$, there were: $12,1,68$ and 43 patients in stages 1, 2, 3 and 4 , respectively. Whereas in the group with a smoking index $\geq 400$, there were: $47,192,234$ and 10 patients in stages $1,2,3$ and 4 , respectively $(p=0.024)$. Details are shown in Table 4.
Table 4. Factors associated with smoking index among smoking ESCC patients, Guangzhou, 2005-2010 ( $\mathrm{N}=944)$

\begin{tabular}{|c|c|c|c|}
\hline Characteristics & $\begin{array}{c}\text { Smoking } \\
\text { index } \\
<100\end{array}$ & $\begin{array}{l}\text { Smoking } \\
\text { index } \\
\geq 100\end{array}$ & $p$ \\
\hline Sample size & 125 & 483 & \\
\hline Gender, M/F & $120 / 5$ & $479 / 4$ & 0.009 \\
\hline Age (years) & $56.4 \pm 10.9$ & $58.4 \pm 8.2$ & 0.028 \\
\hline$A L B, g / L$ & $43.0 \pm 4.4$ & $42.8 \pm 4.1$ & 0.776 \\
\hline GLB, g/L & $26.1 \pm 4.5$ & $26.3 \pm 5.1$ & 0.601 \\
\hline CEA, ng/mL & $3.1 \pm 2.6$ & $3.6 \pm 2.8$ & 0.094 \\
\hline Tumor site, $\mathrm{n}$ & & & 0.705 \\
\hline Upper & 15 & 49 & \\
\hline Middle & 70 & 289 & \\
\hline Distal & 40 & 145 & \\
\hline
\end{tabular}


Table 4. Continued

\begin{tabular}{l|c|c|c|}
\hline Chamacteristics & $\begin{array}{c}\text { Smoking } \\
\text { inder } \\
<100\end{array}$ & $\begin{array}{c}\text { Smoking } \\
\text { inder } \\
\geq 100\end{array}$ & p \\
\hline $\begin{array}{l}\text { Tumor size, cm } \\
\text { Differentiation }\end{array}$ & $4.7 \pm 1.9$ & $4.5 \pm 1.8$ & 0.495 \\
G1 & 17 & 111 & 0.016 \\
G2 & 73 & 218 & \\
G3 & 35 & 154 & \\
TNM, n & & & 0.024 \\
Stage 1 & 12 & 47 & \\
Stage 2 & 68 & 192 & \\
Stage 3 & 43 & 234 & \\
Stage 4 & 2 & 10 &
\end{tabular}

Numbers with \pm represent mean \pm SD

\section{Association of smoking index and clinical outcomes}

We further determined the prognostic impact of smoking index on ESCC patients who smoke. KaplanMeier survival analysis was performed, and results indicated that a smoking index $<400$ was accompanied by a significantly better prognosis, in terms of OS $(p=0.003)$ and favorable DFS $(p=0.032)$ (Figure 2$)$.

Analysis of the independent factors for clinical outcomes of patients with smoking

As shown in Table 5, and in Supplementary file Table 1 , multivariate analysis showed that age $(\mathrm{HR}=1.022$; 95\% CI: 1.009-1.035; $\mathrm{p}<0.001)$, TNM staging $(\mathrm{HR}=2.047 ; 95 \% \mathrm{CI}: 1.706-2.456 ; \mathrm{p}<0.001)$, and smoking index $(\mathrm{HR}=1.070$; 95\% CI: 1.008-1.135;

Figure 2. Survival analyses for revealing the prognostic value of smoking index:

(A) Kaplan-Meier survival analysis was performed and results indicated that smoking index $<400$ were accompanied with significantly better prognosis, in terms of overall survival ( $p=0.003)$; (B) ESCC patients with smoking index $<400$ have favorable disease-free survival $(p=0.032)$

A

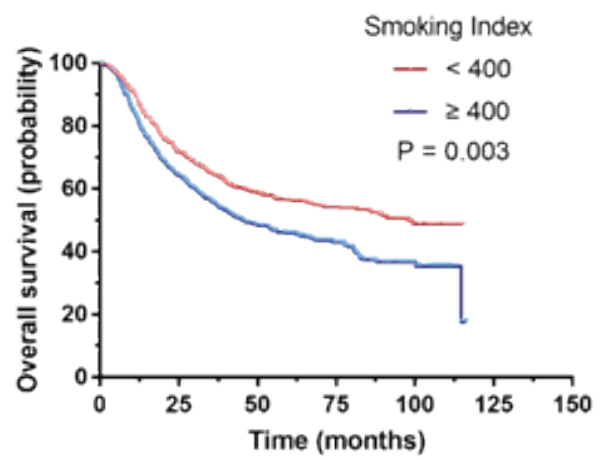

B

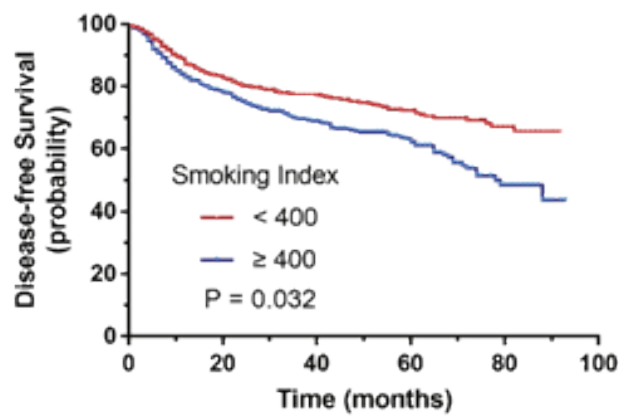

Table 5. Analysis of the independent factors for overall survival among smoking ESCC patients, Guangzhou, 2005-2010 (N=944)

\begin{tabular}{|c|c|c|c|c|c|c|}
\hline \multirow[t]{2}{*}{ Variables } & \multicolumn{3}{|c|}{ Univariate analysis } & \multicolumn{3}{|c|}{ Vultivariate analysis } \\
\hline & IIR & $95 \%$ CI & p & IIR & $95^{\circ} \mathrm{COI}$ & p \\
\hline Gender & 1.825 & 0.839-3.969 & 0.129 & & & \\
\hline Age & 1.016 & $1.003-1.030$ & 0.017 & 1.022 & $1.009-1.035$ & $<0.001$ \\
\hline ALB & 0.983 & $0.991-1.073$ & 0.204 & & & \\
\hline GLB & 1.011 & $0.988-1.033$ & 0.354 & & & \\
\hline CEA & 1.031 & $0.991-1.073$ & 0.127 & & & \\
\hline Tumor site & 0.931 & $0.773-1.122$ & 0.455 & & & \\
\hline Tumor size & 1.065 & $1.003-1.132$ & 0.041 & & & \\
\hline Differentiation & 1.075 & $0.920-1.257$ & 0.363 & & & \\
\hline TNM & 1.964 & $1.627-2.370$ & $<0.001$ & 2.047 & $1.706-2.456$ & $<0.001$ \\
\hline Smoking index & 1.054 & $1.005-1.354$ & 0.004 & 1.070 & $1.008-1.135$ & 0.025 \\
\hline
\end{tabular}


$\mathrm{p}=0.025)$ were independently correlated with OS (Table 5). While for DFS, multivariate analysis showed that only TNM stage (HR=1.435; 95\% CI: 1.159 1.777; $\mathrm{p}=0.001)$ and smoking index $(\mathrm{HR}=1.070$; 95\% CI: 1.008-1.135; $\mathrm{p}=0.025$ ) were independently correlated with DFS (Supplementary file Table 1).

\section{DISCUSSION}

The results of this study show that in patients diagnosed with ESCC in the Cancer Centre, OS was significantly associated with smoking as well as with recognized risk factors including age and TNM stage. In addition, we found that ESCC patients with a higher smoking index had worse clinical outcomes. For all ESCC patients, age, CEA, TNM staging and smoking were independently correlated with OS. While for ESCC patients who smoked, only age, TNM staging and smoking index were identified as independent risk factors correlated with OS. The results show that smoking is not only a risk factor for developing ESCC but also an independent risk factor for poor prognosis. Moreover, we found that the higher the smoking amount, the worse the prognosis.

ESCC is the major type of EC in China ${ }^{22}$. The histological type of ESCC accounted for more than $80 \%$ of EC patients ${ }^{22,23}$. To avoid further bias, the study only included ESCG patients. As EC is one of the most common malignancies, its incidence is generally higher in male than female patients ${ }^{23}$, and the ratio of males to females is about $2: 1$. It is generally believed that EC is a malignant tumor formed by the interaction of multiple factors and multiple genes ${ }^{1,8,10}$. Although many studies have conducted in-depth research on the prevention and treatment of EC and achieved results, the prognosis of EC is poor because of its concealed and invasive nature. Identifying the risk factors for EC and then using risk stratification and different interventions can effectively reduce the mortality associated with EC.

It is well established that smoking is an important factor in the development of $\mathrm{EC}^{10,11}$. The unique chemical compounds of tobacco can regulate the expression of a large number of genes, including genes encoding tumor invasion and metastasis, which can lead to changes in tumor molecular physiology ${ }^{15,16,22}$. However, there is only little evidence of the association between smoking and the prognosis of EC, and the conclusions are inconsistent. Situ et al. ${ }^{24}$ found that smoking before ESCC diagnosis is an independent factor of prognosis. Our study confirmed that smoking is an independent prognostic factor for ESCC. In addition, we confirmed that smoking has an amount-dependent effect on the prognosis of ESCC. The higher the smoking index, the worse the patient's prognosis. However, it is worth noting that the harm of secondhand smoke has been confirmed by many studies ${ }^{25-29}$. In ESCG, there is no research on the relationship between secondhand smoke and EC. How secondhand smoke affects the occurrence and development of EC requires further research and confirmation. The relationship between tobacco and occurrence of ESCC has been confirmed in many studies. However, for the prognosis of patients with esophageal cancer, especially in ESCC, which is a cancer with low incidence in the West, the prognostic impact of tobacco in ESCC still lacks medical evidence. In this study, we demonstrated that tobacco is an independent risk factor for poor prognosis in ESCC patients. The higher smoking index, the worse the prognosis of ESCC patients.

In relation to surgical group, many factors could interfere in the outcome: surgical access (thoracoscopic is related to more respiratory complications than transhiatal approach), number of retrieved lymph nodes (as a marker of sufficient lymphadenectomy), and complications ${ }^{30}$. However, in this study, multivariate analysis suggested that smoking and smoking index were independent risk factors for poor prognosis in ESCG patients. This means that regardless of the surgical access or complications, the smoking and smoking index are still independently affecting the prognosis of ESCC.

In our study, we found that smokers' CEA levels were significantly higher than those of non-smokers. CEA is a classic tumor marker. Studies also found smoking can raise CEA levels ${ }^{31,32}$. Fukuda et al. ${ }^{33}$ showed that CEA-positive patients were more often heavy smokers. This result is consistent with our results. However, the molecular mechanism by which tobacco increases CEA levels remains unclear. In our study, we also found that smokers have lower globulin levels. This is similar to the results of a previous study, which reported that smoking induces changes such as decreased leukocyte chemotaxis, decreased production of globulins, and impaired phagocytosis ${ }^{34}$. Furthermore, our study found that smokers and non- 
smokers have significant differences in the tumor sites. The proportion of esophageal cancer in distal esophagus among smokers was significantly higher than in non-smokers. Moreover, the tumor size of esophageal cancer of smokers is significantly larger than that of non-smokers, as similarly reported ${ }^{35}$. Whether tobacco promotes the proliferation of esophageal cancer cells is an interesting but still unclear question.

Neoadjuvant chemoradiotherapy has been proposed for ESCC patients ${ }^{36}$. A recent study has revealed that neoadjuvant chemoradiotherapy followed by esophagectomy has a significant survival benefit compared to esophagectomy alone ${ }^{37}$. However, following neoadjuvant chemoradiotherapy, a certain proportion of ESCC patients do not respond to chemoradiotherapy ${ }^{38,39}$. Studies have found that neoadjuvant therapy is not associated with better results than surgery alone in ESCC patients ${ }^{40,41}$. However, the patients included in this study did not receive neoadjuvant chemoradiotherapy. But whether smoking has value to predict the benefit after neoadjuvant chemoradiotherapy is a question waiting to be answered.

\section{Strengths and limitations}

The current study has some limitations. First, some other baseline characteristics, variations in therapy regimens, as well as interactions between variables may have caused bias in the results. This study confirms that the smoking and smoking index predicts poor prognosis in ESCG patients after surgery, but further studies are still needed to confirm the relationship between smoking and prognosis in ESCC patients who have not received surgery. Despite this, the results showed a strong possibility for the use of smoking as a predictive marker for ESCC patient prognosis after surgery.

\section{CONCLUSIONS}

The results of the present study showed that smoking is independently associated with OS in ESCC patients. Therefore, smoking may be used as a predictive indicator for pretreatment evaluation and adjustment of treatment regimen.

\section{REFERENCES}

1. Stahl M, Oliveira J. Esophageal cancer: ESMO clinical recommendations for diagnosis, treatment and follow-up. Ann Oncol. 2009;20(Suppl 4):32-33. doi:10.1093/annonc/mdp121

2. Bray F, Ferlay J, Soerjomataram I, Siegel R, Torre L, Jemal A. Global cancer statistics 2018: GLOBOCAN estimates of incidence and mortality worldwide for 36 cancers in 185 countries. CA Cancer J Clin. 2018;68(6):394-424. doi:10.3322/caac. 21492

3. Torre LA, Bray F, Siegel RL, Ferlay J, Lortet-Tieulent J, Jemal A. Global cancer statistics, 2012. CA Cancer J Clin. 2015;65(2):87-108. doi:10.3322/caac.21262

4. Lin Y, Totsuka Y, Shan B, et al. Esophageal cancer in high-risk areas of China: research progress and challenges. Ann Epidemiol. 2017;27(3):215-221. doi:10.1016/j.annepidem.2016.11.004

5. Lin Y, Totsuka Y, He Y, et al. Epidemiology of esophageal cancer in Japan and China. J Epidemiol. 2013;23(4):233242. doi:10.2188/jea.je20120162

6. Jain S, Dhingra S. Pathology of esophageal cancer and Barrett's esophagus. Ann Cardiothorac Surg. 2017;6(2):99-109. doi:10.21037/acs.2017.03.06

7. Okamura A, Watanabe M. [Perioperative Management Team in Esophageal Cancer Surgery]. Kyobu Geka. 2017;70(8):712-715.

8. Tang WR, Chen ZJ, Lin K, Au WW. Development of esophageal cancer in Chaoshan region, China: association with environmental, genetic and cultural factors. Int J Hyg Environ Health. 2015;218(1):12-18. doi:10.1016/j.ijheh.2014.10.004

9. Yang CS, Chen X, Tu S. Etiology and Prevention of Esophageal Cancer. Gastrointest Tumors. 2016;3(1):316. doi:10.1159/000443155

10. Zhang Y. Epidemiology of esophageal cancer. World J Gastroenterol. 2013;19(34):5598-5606. doi:10.3748/wjg.v19.i34.5598

11. Zambon P, Talamini R, La Vecchia C, et al. Smoking, type of alcoholic beverage and squamous-cell oesophageal cancer in northern Italy. Int J Cancer. 2000;86(1):144-149. doi:10.1002/(sici)1097-0215(20000401)86:1<144::aidijc23>3.0.co;2-b

12. Nance R, Delaney J, Mcevoy JW, et al. Smoking intensity (pack/day) is a better measure than packyears or smoking status for modeling cardiovascular disease outcomes. J Clin Epidemiol. 2017;81:111-119. doi:10.1016/j.jclinepi.2016.09.010

13. Huxley RR, Yatsuya H, Lutsey PL, Woodward M, Alonso A, Folsom AR. Impact of age at smoking initiation, dosage, and time since quitting on cardiovascular disease in african americans and whites: the atherosclerosis risk in communities study. Am J Epidemiol. 2012;175(8):816826. doi:10.1093/aje/kwr391

14. Ehteshami-Afshar S, Momenan A, Hajshekholeslami F, Azizi F, Hadaegh F. The impact of smoking status on 9.3 years incidence of cardiovascular and all-cause mortality among Iranian men. Ann Hum Biol. 2014;41(3):249-254. doi:10.3109/03014460.2013.853834 
15. Bartsch H, Malaveille C, Friesen M, Kadlubar FF, Vineis P. Black (air-cured) and blond (flue-cured) tobacco cancer risk. IV: Molecular dosimetry studies implicate aromatic amines as bladder carcinogens. Eur J Cancer. 1993;29A(8):1199-1207. doi:10.1016/s09598049(05)80315-6

16. Rassekh CH. Tobacco cancer of the oral cavity and pharynx. W V Med J. 2001;97(1):8-12.

17. Oze I, Matsuo K, Ito H, et al. Cigarette smoking and esophageal cancer risk: an evaluation based on a systematic review of epidemiologic evidence among the Japanese population. Jpn J Clin Oncol. 2012;42(1):6373. doi:10.1093/jjco/hyr170

18. Sobus SL, Warren GW. The biologic effects of cigarette smoke on cancer cells. Cancer. 2014;120(23):3617-3626. doi:10.1002/cncr.28904

19. Lagergren J, Bergstrom R, Lindgren A, Nyrén O. The role of tobacco, snuff and alcohol use in the aetiology of cancer of the oesophagus and gastric cardia. Int J Cancer. 2000;85(3):340-346. doi:10.1002/(sici)10970215(20000201)85:3<340::aid-ijc8>3.0.co;2-n

20. Yang Y, Huang X, Zhou L, et al. Clinical use of tumor biomarkers in prediction for prognosis and chemotherapeutic effect in esophageal squamous cell carcinoma. BMC Cancer. 2019;19(1):526. doi:10.1186/ s12885-019-5755-5

21. Zheng C, Yan H, Zeng J, Cai S, Wu X. Comparison of pegylated interferon monotherapy and de novo pegylated interferon plus tenofovir combination therapy in patients with chronic hepatitis B. Infect Drug Resist. 2019;12:845854. doi:10.2147/idr.s195144

22. Feng RM, Zong YN, Cao SM, Xu RH. Current cancer situation in China: good or bad news from the 2018 Global Cancer Statistics? Cancer Commun (Lond). 2019;39(1):22. doi:10.1186/s40880-019-0368-6

23. Kou K, Baade PD, Guo X, et al. Area socioeconomic status is independently associated with esophageal cancer mortality in Shandong, China. Sci Rep. 2019;9(1):6388. doi:10.1038/s41598-019-42774-x

24. Situ D, Wang J, Lin P, et al. Do tumor location and grade affect survival in pT2N0M0 esophageal squamous cell carcinoma? J Thorac Cardiovasc Surg. 2013;146(1):4551. doi:10.1016/j.jtcvs.2013.01.034

25. Agaku IT, Odani S, King BA, Armour BS. Prevalence and correlates of secondhand smoke exposure in the home and in a vehicle among youth in the United States. Prev Med. 2019;126:105745. doi:10.1016/j.ypmed.2019.06.003

26. Rahman M, Mostofa MG, Rahman MM, et al. Secondhand smoke and illness: are mother-child pairs from low socio-economic strata at increased risk in Bangladesh? Int J Tuberc Lung Dis. 2019;23(4):412-421. doi:10.5588/ijtld.18.0186

27. Zielen S, Fussbroich D. Impact of secondhand smoke on cystic fibrosis: is there a link to fatty acid metabolism? Thorax. 2019;74(6):529-530. doi:10.1136/ thoraxjnl-2019-213042

28. Cai S, Ou Z, Liu D, et al. Risk factors associated with liver steatosis and fibrosis in chronic hepatitis B patient with component of metabolic syndrome. United European Gastroenterol J. 2018;6(4):558-566. doi: $10.1177 / 2050640617751252$

29. Ou H, Cai S, Liu Y, Xia M, Peng J. A noninvasive diagnostic model to assess nonalcoholic hepatic steatosis in patients with chronic hepatitis B. Therap Adv Gastroenterol. 2017;10(2):207-217. doi:10.1177/1756283x16681707

30. Kataoka K, Takeuchi H, Mizusawa J, et al. Prognostic impact of postoperative morbidity after esophagectomy for esophageal cancer: exploratory analysis of JCOG9907. Ann Surg. 2017;265(6):1152-1157. doi:10.1097/sla.0000000000001828

31. Alexander JC, Silverman NA, Chretien PB. Effect of age and cigarette smoking on carcinoembryonic antigen levels. JAMA. 1976;235(18):1975-1979. doi:10.1001/jama.235.18.1975

32. Sajid KM, Parveen R, Sabih DE, et al. Carcinoembryonic antigen (CEA) levels in hookah smokers, cigarette smokers and non-smokers. J Pak Med Assoc. 2007;57(12):595.

33. Fukuda I, Yamakado M, Kiyose H. Influence of smoking on serum carcinoembryonic antigen levels in subjects who underwent multiphasic health testing and services. J Med Syst. 1998;22(2):89-93. doi:10.1023/a:1022643102208

34. Johannsen A, Susin C, Gustafsson A. Smoking and inflammation: evidence for a synergistic role in chronic disease. Periodontol 2000. 2014;64(1):111-126. doi:10.1111/j.1600-0757.2012.00456.x

35. Su X, Fang D, Li X, et al. The Influence of Tumor Size on Oncologic Outcomes for Patients with Upper Tract Urothelial Carcinoma after Radical Nephroureterectomy. Biomed Res Int. 2016;2016. doi:10.1155/2016/4368943

36. Nygaard K, Hagen S, Hansen HS, et al. Pre-operative radiotherapy prolongs survival in operable esophageal carcinoma: A randomized, multicenter study of preoperative radiotherapy and chemotherapy. The second Scandinavian trial in esophageal cancer. World J Surg. 1992;16:1104-1109. doi:10.1007/bf02067069

37. Gebski V, Burmeister B, Smithers BM, Foo K, Zalcberg J, Simes J. Survival benefits from neoadjuvant chemoradiotherapy or chemotherapy in oesophageal carcinoma: A meta-analysis. Lancet Oncol. 2007;8:226234. doi:10.1016/s1470-2045(07)70039-6

38. Stahl M, Stuschke M, Lehmann N, et al. Chemoradiation with and without surgery in patients with locally advanced squamous cell carcinoma of the esophagus. J Clin Oncol. 2005;23:2310-2317. doi:10.1200/jco.2005.00.034

39. Li SH, Huang EY, Lu HI, et al. Phosphorylated mammalian target of rapamycin expression is associated with the response to chemoradiotherapy in patients with esophageal squamous cell carcinoma. J Thorac Cardiovasc Surg. 2012;144:1352-1359. doi:10.1016/j. jtcrs.2012.06.049 
40. Steyerberg EW, Neville BA, Koppert LB, et al. Surgical mortality in patients with esophageal cancer: Development and validation of a simple risk score. J Clin Oncol. 2006;24:4277-4284. doi:10.1200/jco.2005.05.0658

41. Mota FC, Cecconello I, Takeda FR, Tustumi F, Sallum RAA, Bernardo WM. Neoadjuvant therapy or upfront surgery? A systematic review and meta-analysis of T2N0 esophageal cancer treatment options. Int J Surg. 2018;54:176-181. doi:10.1016/j.ijsu.2018.04.053

ACKNOWLEDGEMENTS

We thank Weidong Wang for his helpful assistance in the study.

CONFLICTS OF INTEREST

The authors have completed and submitted the ICMJE Form for Disclosure of Potential Conflicts of Interest and none was reported.

\section{FUNDING}

The study was funded by the National Natural Science Foundation of China (No. 81802713).

PROVENANCE AND PEER REVIEW

Not commissioned; externally peer reviewed. 